OPEN ACCESS

Edited by:

Hua-Bin Li,

Sun Yat-sen University, China

Reviewed by:

Anca loana Nicolau,

Dunarea de Jos University, Romania

Steven W. Polyak,

University of South Australia,

Australia

${ }^{*}$ Correspondence:

Zhanli Wang

wang.zhanli@hotmail.com

tThese authors have contributed equally to this work

Specialty section: This article was submitted to Antimicrobials, Resistance and

Chemotherapy,

a section of the journal

Frontiers in Microbiology

Received: 15 January 2019

Accepted: 20 May 2019

Published: 06 June 2019

Citation:

Yu H, Liu M, Liu Y, Qin L, Jin M and

Wang Z (2019) Antimicrobial Activity

and Mechanism of Action of

Dracocephalum moldavica $L$. Extracts Against Clinical Isolates of Staphylococcus aureus.

Front. Microbiol. 10:1249.

doi: 10.3389/fmicb.2019.01249

\section{Antimicrobial Activity and Mechanism of Action of Dracocephalum moldavica $L$. Extracts Against Clinical Isolates of Staphylococcus aureus}

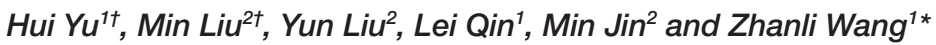 \\ 'The Second Affiliated Hospital, Baotou Medical College, Baotou, China, ${ }^{2}$ School of Public Health, Baotou Medical College, \\ Baotou, China
}

Background: Dracocephalum moldavica L. is a popular traditional medicine used by many countries, which has a wide range of pharmacological effects. The aim of this work was to investigate the antimicrobial effects of $D$. moldavica $L$. extracts against clinical isolates of Staphylococcus aureus. Our results demonstrated that the minimal inhibitory concentration (MIC) for 50 and $90 \%$ of $S$. aureus isolates $\left(\mathrm{MIC}_{50}\right.$ and $\left.\mathrm{MIC}_{90}\right)$ of the ethyl acetate (EtOAc) fraction from D. moldavica $L$. ethanol extract were 780 and 1,065 $\mu \mathrm{g} / \mathrm{ml}$, respectively. We further observed that the EtOAc fraction disrupted 24-h biofilm caused cell membrane damage and irregular cell shape. Additionally, the EtOAc fraction showed slight to moderate toxic effects on human epidermal keratinocyte (HaCaT) cells. Moreover, the results of the differential proteome revealed that 231 proteins were upregulated, while 61 proteins were downregulated in S. aureus after treatment with the EtOAc fraction. The differentially expressed proteins were functionally categorized by the Gene Ontology (GO) enrichment and Kyoto Encyclopedia of Genes and Genomes (KEGG) pathway. These proteins contribute to membrane damage, inhibition of biofilm formation, and changes in energy metabolism. Thus, the EtOAc fraction of $D$. moldavica $L$. ethanol extract, as a natural product, has the potential to be used as an antimicrobial agent to control the clinical isolates of $S$. aureus.

Keywords: medicinal plant, antimicrobial, cytotoxicity, proteomics, differentially expressed protein, biofilm

\section{INTRODUCTION}

Staphylococcus aureus is a serious human pathogen known to cause numerous bacterial infections at the level of the bloodstream, lower respiratory tract, and skin and soft tissue (Sakr et al., 2018). Penicillin was initially highly effective for treatment of S. aureus infections. However, the widespread use of the penicillin led to the emergence of penicillin-resistant $S$. aureus (PRSA) (Chen and Huang, 2014). With the release of beta-lactamase-resistant penicillins such 
as methicillin and oxacillin in the 1970s, methicillin-resistant S. aureus (MRSA) emerged and became an important cause of infectious diseases acquired in hospitals and communities worldwide (McDonald et al., 1981). Since this situation exists, it is necessary to fight against MRSA. Moreover, many recent studies showed that MRSA spread along the food chain, indicating urgent need for control programs to avoid food transmission (Doulgeraki et al., 2017; Oniciuc et al., 2017; Li et al., 2018). However, the treatment of MRSA infections is complicated due to its multi-drug resistance.

Traditional antibiotics are gradually loosing their efficacy against many bacterial pathogens due to fast development of antibiotic resistance in microorganisms. Glycopeptides and several newer classes of antibiotics have proven to have activity against MRSA, including linezolid of the oxazolidinone class and daptomycin of the lipopeptide class (Kali, 2015). However, the drugs available for clinical treatment of MRSA are very limited. Therefore, there is an urgent demand for novel antimicrobial agents able to replace the antimicrobial activity of old antibiotics. Several studies have shown that extracts from plant species may be active against multi-drug resistant bacteria, including MRSA (Martin and Ernst, 2003).

Dracocephalum moldavica $L$. is a medicinal plant used by many countries, which has a wide range of pharmacological effects, including anti-inflammatory, antioxidative, and cardioprotective effects (Jiang et al., 2014). D. moldavica L. has been reported to contain steroid, flavonoids, glycoside, saponins, tannins, and phenolic compounds (Zhang et al., 2018). The aim of this work was to investigate the antimicrobial and cytotoxic effects of D. moldavica L. extracts. Moreover, the antimicrobial mechanism was also investigated.

\section{MATERIALS AND METHODS}

\section{Plant Material and Extracts Preparation}

D. moldavica L. was collected in the county of Tongliao (latitude $43^{\circ} 39^{\prime}$ north and longitude $122^{\circ} 14^{\prime}$ east), Inner Mongolia, China. The plant material was air dried in shade at room temperature for 7-14 days and ground into powder using an electric grinder (Da Xiang, China). Then, the powdered material $(50 \mathrm{~g})$ was subjected to extraction twice with $500 \mathrm{ml}$ of $65 \%$ ethanol at a temperature of $60^{\circ} \mathrm{C}$ for $120 \mathrm{~min}$. The supernatants were then filtered, gathered, and concentrated under vacuum in a rotary evaporator (Yarong RE-2000A, China) to remove ethanol. Finally, the ethanol extract was subjected to extraction with petroleum ether (petrol), dichloromethane $\left(\mathrm{CH}_{2} \mathrm{Cl}_{2}\right)$, ethyl acetate (EtOAc), and n-butyl alcohol $(\mathrm{n}-\mathrm{BuOH})$ using separating

\footnotetext{
Abbreviations: $\mathrm{CH}_{2} \mathrm{Cl}_{2}$, Dichloromethane; DMEM, Dulbecco's modified Eagle's medium; DMSO, Dimethyl sulfoxide; EtOAc, Ethyl acetate; FDR, False discovery rate; GO, Gene Ontology; HaCaT, Human epidermal keratinocyte; HPLC-MS, Highperformance liquid chromatography-mass spectrometry; KEGG, Kyoto Encyclopedia of Genes and Genomes; MHB, Mueller-Hinton Broth; MIC, Minimal inhibitory concentration; MRSA, Methicillin-resistant Staphylococcus aureus; MSSA, Methicillinsensitive Staphylococcus aureus; MTT, 3-(4,5-dimethyl-2-thiazolyl)-2, 5-diphenyl2H-tetrazolium bromide; PRSA, Penicillin-resistant Staphylococcus aureus.
}

funnels. The resultant fractions were evaporated, dried, and stored at $-20^{\circ} \mathrm{C}$ for further experiments.

\section{High-Performance Liquid Chromatography (HPLC) Analysis}

The separation was carried out on a HPLC system (Shimadzu LC-20AT) with a diode array detector (DAD) equipped with Inertsil ODS-SP column $(250 \times 4.6 \mathrm{~mm}, 5 \mu \mathrm{m}$ particle size $)$. Mobile phase A was acetonitrile, and mobile phase B were ultrapure water and acetic acid (999/1, v/v). Gradient conditions were as follows: $10-20 \mathrm{~min}$, linear gradient $70-55 \% \mathrm{~A}$ in B; 20-21 min, linear gradient $55-70 \% \mathrm{~A}$ in $\mathrm{B} ; 21-31 \mathrm{~min}$, isocratic $70-70 \% \mathrm{~A}$ in $\mathrm{B}$. The injection volume was $10 \mu \mathrm{l}$. The flow rate was $1.0 \mathrm{ml} / \mathrm{min}$. For qualitative analysis, the fractions were evaluated with the standards (tallianine, rosmarinic acid, luteolin, apigenin, and diosmetin) as the references.

\section{Bacterial Strains and Antibiotic Susceptibility}

The following Gram-positive, Gram-negative bacteria, and fungi strains from the Second Affiliated Hospital of Baotou Medical College were employed: S. aureus (120 isolates), Staphylococcus epidermidis (6 isolates), Staphylococcus haemolyticus (5 isolates), Enterococcus faecalis (7 isolates), Enterococcus faecium (7 isolates), Klebsiella pneumonia (5 isolates), Escherichia coli, (10 isolates), Acinetobacter baumannii (6 isolates), Pseudomonas aeruginosa (9 isolates), Saccharomyces albicans (7 isolates), Candida glabrata (5 isolates), Candida krusei (5 isolates), and Candida parapsilosis (3 isolates). S. aureus isolates comprised of $23 \mathrm{MRSA}$ and 97 methicillin-sensitive $S$. aureus (MSSA). The clinical isolates used in this study were maintained at $-80^{\circ} \mathrm{C}$ in a freezer. Prior to assay, bacteria and fungi strains were grown overnight at 37 and $35^{\circ} \mathrm{C}$, respectively. The isolates were tested for antibiotic susceptibility using BD Phoenix 100 automated system and the conventional Kirby-Bauer agar diffusion disk method as recommended by the Clinical and Laboratory Standards Institute (Clinical and Laboratory Standards Institute, 2018).

\section{Evaluation of the Antimicrobial Activity}

Disk diffusion assays were performed in triplicate for D. moldavica L. extracts. Antibacterial and antifungal activities were determined on Mueller Hinton agar and Sabouraud dextrose agar (Oxoid, UK), respectively. Four 6-mm-diameter disks were placed onto each agar plate and then injected with several dilutions of $D$. moldavica L. extracts $(0,50,100$, and $200 \mu \mathrm{g} /$ $\mathrm{ml}$ ) with a volume of $10 \mu \mathrm{l}$ individually. The plates were incubated at temperatures of $37^{\circ} \mathrm{C}$ for bacteria for $24 \mathrm{~h}$ and $35^{\circ} \mathrm{C}$ for fungi for $24 \mathrm{~h}$. Subsequently, the diameter of inhibition zone surrounding the disk (including the disk) was measured. S. aureus ATCC 25923 was used as a quality control strain. The antibacterial activity of EtOAc fraction of D. moldavica $L$. ethanol extract was further evaluated by minimal inhibitory concentration (MIC). A 2-fold broth-dilution method was utilized to assess MIC. The bacterial population was exposed to several dilutions of EtOAc fraction and incubated at $37^{\circ} \mathrm{C}$ for $16 \mathrm{~h}$. An ELISA reader was used to detect MIC at $595 \mathrm{~nm}$. 
The MIC of EtOAc fraction which inhibits 50 and $90 \%$ of the isolates was denoted $\mathrm{MIC}_{50}$ and $\mathrm{MIC}_{90}$, respectively.

\section{Biofilm Formation Assay}

S. aureus biofilms were allowed to form in the media (TSB $+1 \%$ glucose) at $37^{\circ} \mathrm{C}$ for $24 \mathrm{~h}$ in a 6-well plate. After removal of the medium, the biofilms were washed five times with PBS. EtOAc fraction of $D$. moldavica $L$. ethanol extract was then added to the mature biofilms in the media (TSB $+1 \%$ glucose). The plates were incubated at $37^{\circ} \mathrm{C}$ during $24 \mathrm{~h}$, and then the medium and non-adherent cells were removed by washing with sterile PBS. The effect of EtOAc fraction on biofilm formation was determined using a standard 3-(4,5-dimethyl-2-thiazolyl)-2, 5-diphenyl-2H-tetrazolium bromide (MTT) reduction assay as described previously by Jia et al. (Jia et al., 2011). Ciprofloxacin $(50 \mu \mathrm{g} / \mathrm{ml})$ was used as positive control.

\section{Scanning Electron Microscopy}

In order to observe bacterial morphology under treatment of EtOAc fraction of $D$. moldavica $L$. ethanol extract via a scanning electron microscopy analysis, $S$. aureus was cultured in $60 \mathrm{ml}$ of Mueller-Hinton Broth (MHB) overnight with or without EtOAc fraction treatment. Cells were harvested by centrifugation and washed three times with $0.1 \mathrm{M}$ sodium phosphate buffer solution. Aliquots $(20 \mu \mathrm{l})$ of $S$. aureus suspensions were deposited onto micro cover glass slides for $30 \mathrm{~min}$ and then were immersed into $50 \mathrm{ml}$ of a fixative solution containing $2.5 \%$ glutaraldehyde (Electron Microscopy Sciences, Hatfield, PA, USA) for $30 \mathrm{~min}$. The rest of the procedure was as described previously by Suo et al. (Suo et al., 2018). Morphological alterations of the bacteria were observed and photographed using a Quanta 200 FEG environmental scanning electron microscope (FEI Co., Inc., Hillsboro, OR, USA).

\section{Detection of Cell Membrane Damage}

The cell membrane damage caused by EtOAc fraction of D. moldavica $L$. ethanol extract was determined using a phosphorous leakage method. In briefly, bacteria were harvested at the end of logarithmic phase by centrifugation and resuspended in the $5 \mathrm{ml}$ HEPES-Na buffer ( $5 \mathrm{mM}$, pH 7.0). Bacterial suspensions were added with $5 \mu \mathrm{l}$ of the extract dissolved in dimethyl sulfoxide (DMSO) at final concentration of $300 \mu \mathrm{g} / \mathrm{ml}$. DMSO was used as negative control. The samples were then incubated at $37^{\circ} \mathrm{C}$ for $1,2,3,5,10,15,20,25$, $30 \mathrm{~h}$, respectively. Then the total phosphate was determined as described previously by Luo et al. (Luo et al., 2015).

\section{Cytotoxicity Evaluation}

The in vitro cytotoxicity of EtOAc fraction of $D$. moldavica $L$. ethanol extract was investigated using human epidermal keratinocyte (HaCaT) cell line as described previously by Wadhwa et al. (Wadhwa et al., 2019). HaCaT cells were cultivated in Dulbecco's modified Eagle's medium (DMEM) medium supplemented in 10\% fetal bovine serum (FBS, Thermo Fisher Scientific, MA, USA), streptomycin $(100 \mu \mathrm{g} / \mathrm{ml}$, Thermo Fisher Scientific, MA, USA), and penicillin (100 IU/ml, AppliChem GmbH, Darmstadt, Germany) in a humidified atmosphere containing $5 \% \mathrm{CO}_{2}$ at $37^{\circ} \mathrm{C}$. EtOAc fraction was dissolved in distilled DMSO and DMEM supplemented with $2 \%$ FBS. From the stock solution $(1 \mathrm{mg} / \mathrm{ml})$, serial two-fold dilutions $(0-100 \mu \mathrm{g} / \mathrm{ml})$ were prepared for analysis. Cells were seeded in a 96-well plate at $1 \times 10^{5}$ cells per well. After $24 \mathrm{~h}$, cells were treated with serial dilutions of EtOAc fraction and incubated for $24 \mathrm{~h}$. After incubation, $0.5 \mathrm{mg} / \mathrm{ml}$ of MTT was added to each well for $2 \mathrm{~h}$. Absorbance was measured at $590 \mathrm{~nm}$ using a microplate reader.

\section{Peptide Separation and Identification by Non-Labeled HPLC-MS}

Cells were harvested by centrifugation of $5 \mathrm{ml}$ of bacterial culture. After triple washing with PBS, the bacterial pellet was resuspended in lysis buffer $(2 \%$ SDS, $7 \mathrm{M}$ urea, $1 \times$ Protease Inhibitor Cocktail), followed by sonication on ice for $30 \mathrm{~min}$. Then, samples were centrifuged at $15,000 \mathrm{rpm}$ for $15 \mathrm{~min}$ at $4^{\circ} \mathrm{C}$. The supernatant was used for the measurement of protein concentration with a BCA Assay Kit (Sigma-Aldrich, St. Louis, MO, USA). The sample was then digested to produce the final trypsinized peptide sample as described previously by Suo et al. (Suo et al., 2018). The fraction was separated by Easy-nLC 1000 system (Thermo Fisher Scientific, MA, USA) and analyzed by Q-Exactive mass spectrometer (Thermo Fisher Scientific, MA, USA) equipped with an online nano-electrospray ion source. Peptide sample was loaded onto the trap column (Thermo Scientific Acclaim PepMap C18, $100 \mu \mathrm{m} \times 2 \mathrm{~cm}$ ), with a flow of $10 \mu \mathrm{l} / \mathrm{min}$ for $3 \mathrm{~min}$ and subsequently separated on the analytical column (Acclaim PepMap C18, $75 \mu \mathrm{m} \times 15 \mathrm{~cm}$ ) with a linear gradient, from 3 to $32 \% \mathrm{D}$ in $120 \mathrm{~min}$. The column flow rate was maintained at $300 \mathrm{nl} / \mathrm{min}$. The electrospray voltage of $2 \mathrm{kV}$ versus the inlet of the mass spectrometer was used. The mass spectrometer was run under data dependent acquisition mode and automatically switched under MS and MS/MS mode. MS spectra were acquired across a mass range of $350-1550 \mathrm{~m} / \mathrm{z}$. The dynamic exclusion time was set as $20 \mathrm{~s}$.

\section{Database Search, Protein Identification, and Expression Quantification}

Spectrograms were used for protein identification using PEAKS Studio version 8.0 (Bioinfor Inc. CA) by querying the UniProt database, which contains 9781 protein sequences. Peptides were filtered by $0.01 \%$ false discovery rate (FDR). PEAKS Q was used for peptide and protein abundance calculation. Normalization was performed on averaging the abundance of all peptides. Different expressed proteins were filtered if their $\log 2$ fold change $>2.0$ fold and statistical $p<0.01$. The Gene Ontology (GO) analysis was performed using BLAST2GO version 4 . Whole protein sequence database was analyzed by BlastP using whole database and mapped, annotated with gene ontology database. Statistically altered functions of different expressed proteins were calculated by Fisher's exact test in BLAST2GO. Kyoto Encyclopedia of Genes and Genomes (KEGG) pathway analysis was processed by $\mathrm{KOBAS}^{1}$. Pathways with $p<0.05$ were recognized as significant changed.

\footnotetext{
${ }^{1}$ http://kobas.cbi.pku.edu.cn/
} 


\section{Statistical Analysis}

Statistical analysis was performed with SPSS20.0 software. Analysis of variance (ANOVA) and Tukey multiple range test were used to test the difference. Values of $p<0.05$ were considered statistically significant.

\section{RESULTS}

\section{Antimicrobial Activity of D. moldavica L. Extracts}

Four types of fractions from $D$. moldavica $L$. ethanol extract were obtained: petrol, $\mathrm{CH}_{2} \mathrm{Cl}_{2}$, EtOAc, and $\mathrm{n}-\mathrm{BuOH}$. The antimicrobial activities of the fractions of $D$. moldavica L. were evaluated by the agar diffusion test. In this test, disks containing increasing extract concentrations are placed on an agar plate where organisms have been placed. The EtOAc fraction showed significant antibacterial activity against five Gram-positive bacteria (S. aureus, S. epidermidis, S. haemolyticus, E. faecalis, and E. faecium) in a dose-dependent manner. However, no antimicrobial activity against Gram-negative bacteria and fungi tested was seen (concentration up to $250 \mathrm{mg} / \mathrm{ml}$ ). Additionally, no antimicrobial activities against both Gram-positive and Gram-negative bacteria and fungi were observed for petrol, $\mathrm{CH}_{2} \mathrm{Cl}_{2}$, and $\mathrm{n}-\mathrm{BuOH}$ fractions of D. moldavica $L$. (concentration up to $250 \mathrm{mg} / \mathrm{ml}$ ). Moreover, our results found that the MIC values of the EtOAc fraction for S. aureus isolates ranged from 390 to $1,560 \mu \mathrm{g} / \mathrm{ml}$ with an $\mathrm{MIC}_{50}$ of $780 \mu \mathrm{g} / \mathrm{ml}$ and an $\mathrm{MIC}_{90}$ of $1,065 \mu \mathrm{g} / \mathrm{ml}$.

\section{Phytochemical Analysis of EtOAc Fraction of $D$. moldavica $L$. Ethanol Extract}

Through HPLC analysis of the EtOAc fraction, several compounds were identified. The majority compounds found in the EtOAc fraction were rosmarinic acid (peak 1), tilianin (peak 2), and an unknown compound (peak 4) (Figure 1). The chromatograms also showed that luteolin (peak 3), apigenin (peak 5), and disometin (peak 6) were found in the EtOAc fraction (Figure 1).

\section{Effect of EtOAc Fraction of $D$. moldavica $L$. Ethanol Extract on Membrane Integrity}

The cell membrane integrity of MRSA isolates was measured by UV-absorbing release materials (Figure 2). Compared with control group, the release of phosphate ions from EtOAc fraction group was remarkably increased $(p<0.05)$. The significant difference in increase of phosphate ion leakage was observed within $3 \mathrm{~h}$, and the levels of phosphate ion leakage were stable during treatment of EtOAc fraction of D. moldavica L. ethanol extract for $10 \mathrm{~h}$.

\section{Effect of EtOAc Fraction of $D$. moldavica $L$. Ethanol Extract on Bacterial Morphology}

Changes in the bacterial morphology of MRSA isolates after exposure to EtOAc fraction were observed by scanning electron microscopy analysis. The untreated bacteria were used as the

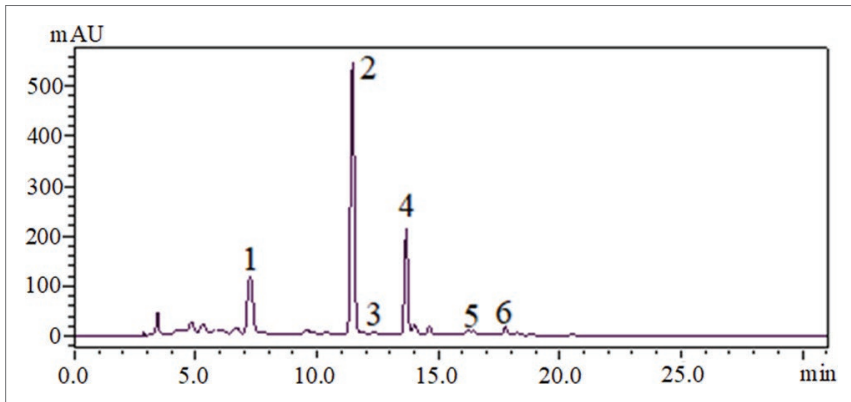

FIGURE 1 | HPLC-DAD chromatograms of EtOAc fraction of $D$. moldavica $L$. ethanol extract, visualized at $330 \mathrm{~nm}$. 1, rosmarinic acid; 2, tilianin; 3, luteolin; 4, an unknown compound; 5, apigenin; 6, disometin.

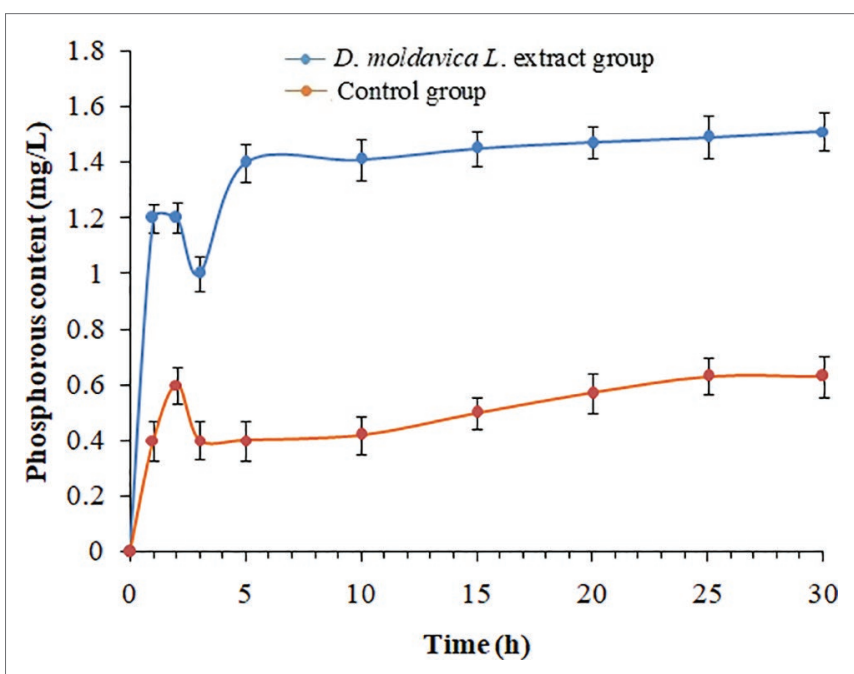

FIGURE 2 | Phosphorous leakage of $S$. aureus treated with and without EtOAc fraction of $D$. moldavica $L$. ethanol extract at different time.

control. As shown in Figure 3, the untreated bacteria displayed a smooth and intact surface with typical morphology. After incubation with EtOAc fraction of D. moldavica L. ethanol extract, bacteria showed the leakage of bacterial content, leading to dramatic changes in morphology. Clearly, these cells were smaller in size than controls. Lysed cells and debris were also observed.

\section{Effect of EtOAc Fraction of $D$. moldavica $L$. Ethanol Extract on Biofilm Formation}

EtOAc fraction of $D$. moldavica $L$. ethanol extract exhibited pronounced dose-dependent inhibitory effects on biofilm formation of tested MRSA strain (Figure 4). EtOAc fraction at doses of 50,100 , and $200 \mu \mathrm{g} / \mathrm{ml}$ was able to inhibit formation of S. aureus $24-\mathrm{h}$ biofilms by 27,31 , and $63 \%$, respectively, as compared to the untreated control.

\section{Effect of EtOAc Fraction of $D$. moldavica $L$. Ethanol Extract on Cytotoxicity}

The cytotoxicity of EtOAc fraction was carried out using MTT assay. The results of $\mathrm{HaCaT}$ cell line treated with 

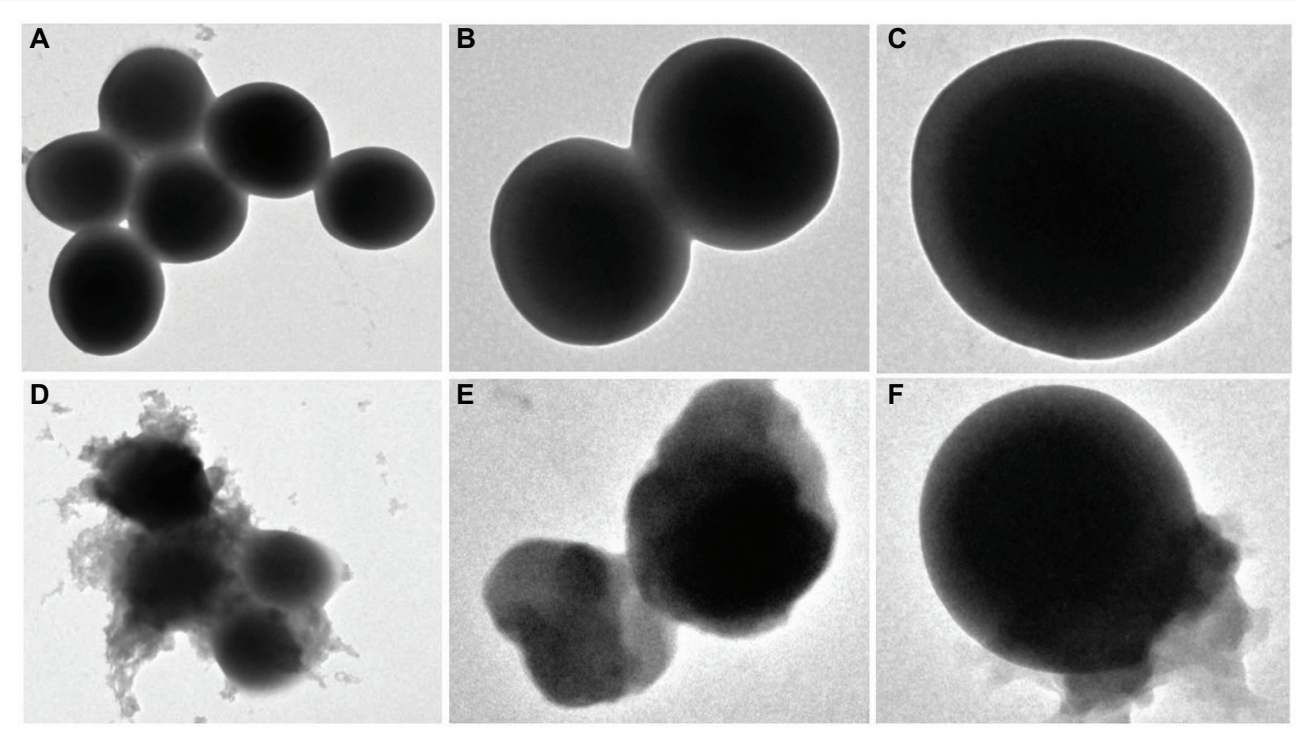

FIGURE 3 | Scanning electron microscopy analysis of S. aureus treated with and without EtOAc fraction of $D$. moldavica L. ethanol extract. Images (A) and (D) were observed at an instrumental magnification of 10,000x; images (B) and (E) at an instrumental magnification of 20,000x; images (C) and (F) at an instrumental magnification of 60,000×. (A-C) D. moldavica L. extract group. (D-F) Control group.

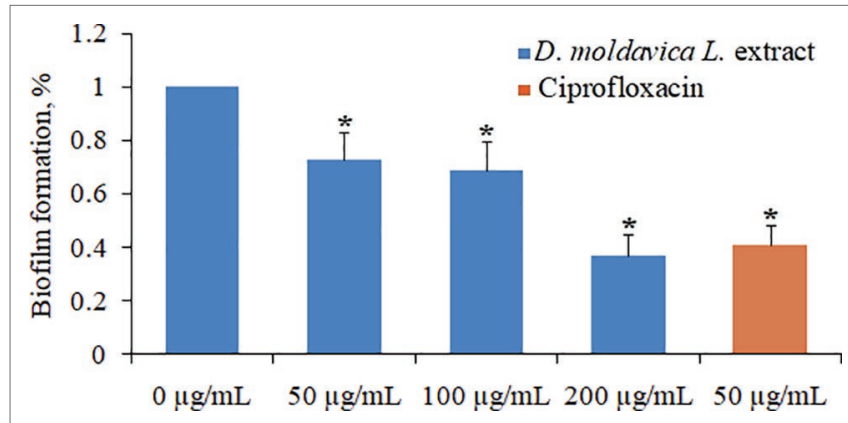

FIGURE 4 | Effect of EtOAc fraction of $D$. moldavica $L$. ethanol extract on biofilm formation of $S$. aureus at concentrations of $0-200 \mu \mathrm{g} / \mathrm{ml}$. * $D$. moldavica $L$. extract-treated group vs. untreated group, $p<0.05$ were considered significant.

TABLE 1 | In vitro cytotoxicity of $D$. moldavica $L$. extract.

\begin{tabular}{lc}
\hline Concentration $(\boldsymbol{\mu g} / \mathbf{m l})$ & $\%$ Inhibition \\
\hline 0 & 0.00 \\
20 & $6.03 \pm 0.39$ \\
40 & $9.17 \pm 0.41$ \\
60 & $17.79 \pm 0.97$ \\
80 & $25.13 \pm 0.73$ \\
100 & $33.27 \pm 0.71$ \\
\hline
\end{tabular}

different concentrations of EtOAc fraction are presented in Table 1. The EtOAc fraction at doses of 20, 40, 60, 80, and $100 \mu \mathrm{g} / \mathrm{ml}$ was able to inhibit proliferation of $\mathrm{HaCaT}$ cell line by $(6.03 \pm 0.39),(9.17 \pm 0.41),(17.79 \pm 0.97)$, $(25.13 \pm 0.73)$, and $(33.27 \pm 0.71) \%$, respectively.

\section{Effect of EtOAc Fraction of $D$. moldavica $L$. Ethanol Extract on Protein Expression}

Based on the MS analysis of each sample, changes in the protein profile were analyzed. The present study showed that 292 proteins exhibited a difference (fold changes $\geq 2$ ) with a FDR of less than $0.01 \%$ (Figure 5A). Among 292 differentially expressed proteins, 231 proteins were significantly upregulated, while 61 proteins were significantly downregulated. Figure 5B shows the volcano of differentially expressed proteins. The top 20 upregulated proteins included undecaprenyl-diphosphatase, delta-hemolysin, and choline dehydrogenase (Table 2). The top 20 downregulated proteins were listed in Table 3, including copper chaperone copZ, antiholin-like protein $\operatorname{lrgB}$, and sitespecific tyrosine recombinase.

\section{Functional Categorization of Differentially Expressed Proteins}

Next, we classified the upregulated and downregulated proteins based on the relative categories annotated by the GO method (Figure 6). Among the differentially expressed proteins classified as being involved in biological process, 140, 201, and 118 proteins were classified into the categories of single-organism process (GO: 0044699; $p=3.98 \mathrm{E}-18$ ), metabolic process (GO: 0008152; $p=2.29 \mathrm{E}-16$ ), and singleorganism metabolic process (GO: 0044710; $p=3.02 \mathrm{E}-16$ ), respectively. For molecular function, 187, 47, and 24 proteins were classified into the categories of catalytic activity (GO: $0003824 ; p=1.18 \mathrm{E}-18$ ), oxidoreductase activity (GO: 0016491; $p=1.46 \mathrm{E}-09$ ), and cofactor binding (GO: 0048037; $p=1.80 \mathrm{E}-06)$, respectively, which were catalytic activity and binding related. Moreover, for cellular component, a majority were intracellular related. 

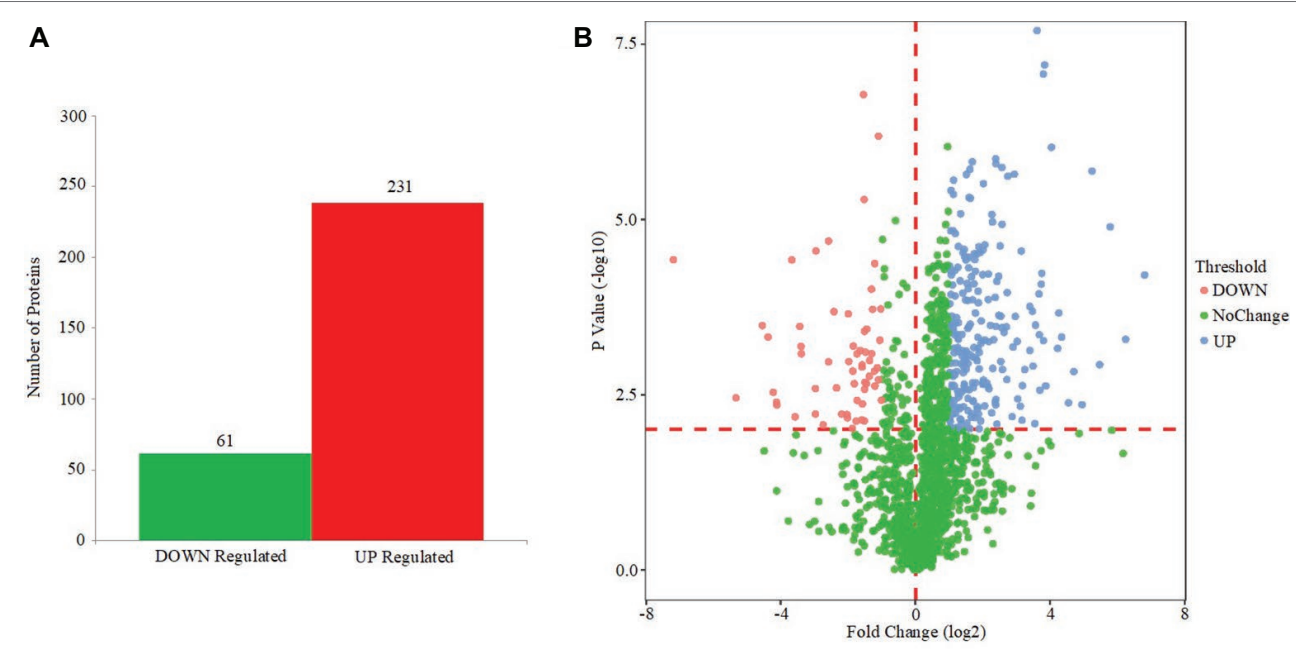

FIGURE 5 | Effect of EtOAc fraction of D. moldavica L. ethanol extract on protein expression. (A) Identification of differentially expressed proteins in S. aureus following EtOAc fraction treatment. (B) The volcano of differentially expressed proteins.

TABLE 2 | Top 20 increased expressed proteins in D. moldavica L. extract treatment group compared to control.

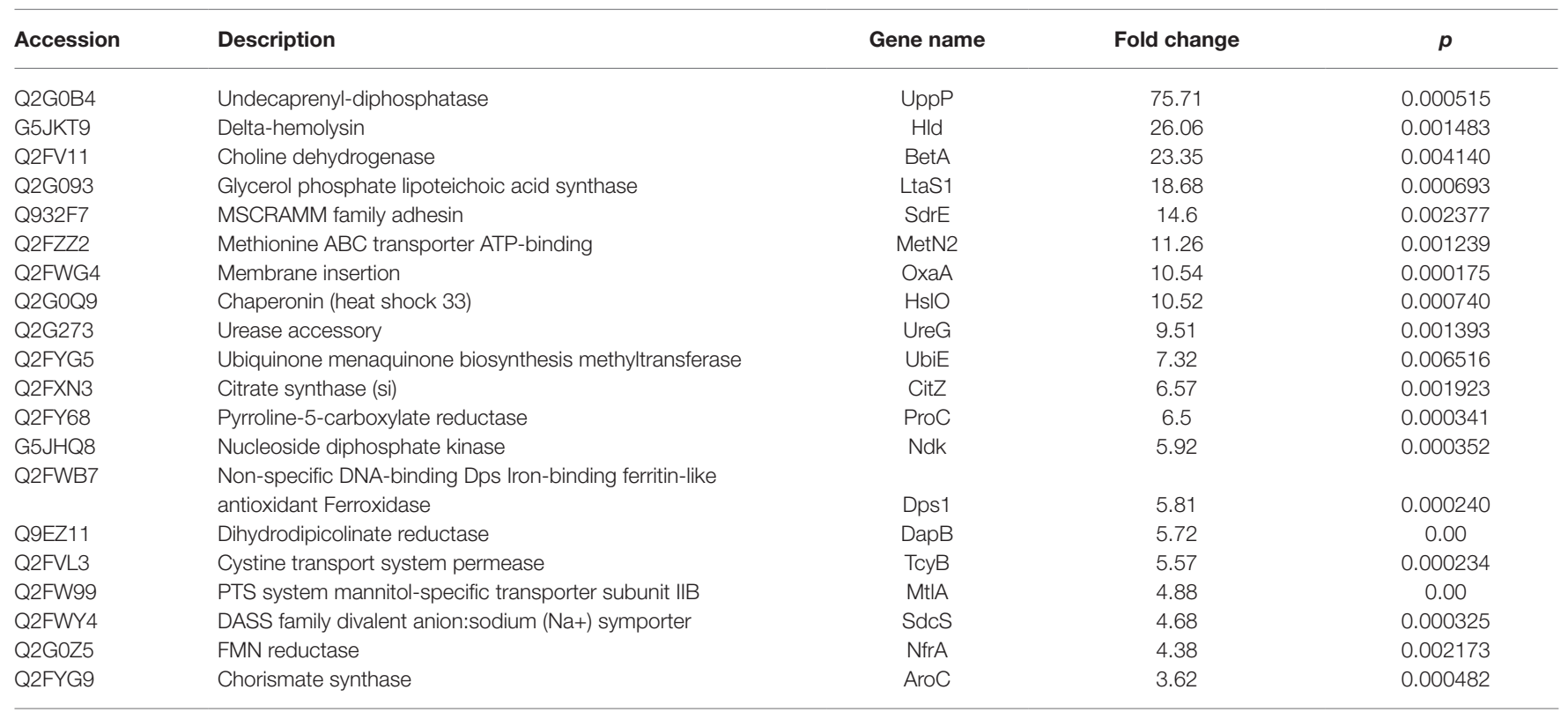

\section{KEGG Pathway Analysis of Differentially Expressed Proteins}

The functions of the differentially expressed proteins were further analyzed by the KEGG pathway annotation method and 39 pathways were obtained (Figure 7). Only two enriched pathways had a $p$ of less than 0.05: microbial metabolism in diverse environments $(p=0.029)$, and fructose and mannose metabolism $(p=0.031)$.

\section{DISCUSSION}

MRSA has become an increasingly important pathogen in hospitals and the community (Campanile et al., 2009). Current antibiotic treatment of MRSA infection has become limited due to the emergence of multidrug-resistant isolates. D. moldavica $L$. is one of the most widely used medicinal plants. To date, however, it is still not known whether D. moldavica $L$. has antimicrobial activity. In the study, we investigated the antimicrobial effects of D. moldavica L. extracts.

The preliminary antibacterial activity results showed that the EtOAc fraction of D. moldavica L. ethanol extract exhibited remarkable antibacterial activity against MRSA isolates with an $\mathrm{MIC}_{50}$ of $780 \mu \mathrm{g} / \mathrm{ml}$ and an $\mathrm{MIC}_{90}$ of $1,065 \mu \mathrm{g} / \mathrm{ml}$. Additionally, agar diffusion test revealed that this fraction exhibited significant antibacterial activity in a dose-dependent manner, indicating that the EtOAc fraction of D. moldavica L. showed promising capacity as new phytotherapeutic candidate against MRSA. 
TABLE 3 | Top 20 decreased expressed proteins in D. moldavica L. extract treatment group compared to control.

\begin{tabular}{|c|c|c|c|c|}
\hline Accession & Description & Gene name & Fold change & $p$ \\
\hline Q2FV63 & Copper chaperone CopZ & CopZ & -40.38 & 0.003516 \\
\hline P60643 & Antiholin-like protein LrgB & $\operatorname{LrgB}$ & -17.51 & 0.004055 \\
\hline Q2FZ30 & Site-specific tyrosine recombinase & XerC & -11.92 & 0.006561 \\
\hline Q931E9 & Surface SAV2496 SAV2497 & SasG & -10.49 & 0.000826 \\
\hline Q2FXV6 & tRNA (5-methylaminomethyl-2-thiouridylate)- methyltransferase & TrmU & -7.86 & 0.002588 \\
\hline Q2G1D7 & Pyruvate formate-lyase activating enzyme & $\mathrm{PflA}$ & -4.09 & 0.006067 \\
\hline Q2FX12 & Low molecular weight tyrosine phosphatase & YfkJ & -3.99 & 0.000223 \\
\hline Q2FVM4 & Respiratory nitrate reductase gamma chain & Narl & -3.66 & 0.009594 \\
\hline Q2FY19 & Zinc uptake regulation ZUR & Zur & -3.63 & 0.001459 \\
\hline Q2FVM2 & Respiratory nitrate reductase beta chain & $\mathrm{NarH}$ & -3.60 & 0.000643 \\
\hline Q2G2U1 & Sensor histidine kinase & Saes & -3.53 & 0.002208 \\
\hline Q2G2M7 & Serine acetyltransferase & CysE & -3.39 & 0.007534 \\
\hline Q2G0B7 & Lysine decarboxylase family & YvdD & -3.12 & 0.000743 \\
\hline Q2FWD8 & 50 S ribosomal L31 type B & RpmE & -3.01 & 0.001282 \\
\hline Q2G1D8 & Formate acetyltransferase & PflB & -2.88 & 0.00 \\
\hline Q2G0Q0 & Pyridoxine biosynthesis amidotransferase & $P d x T$ & -2.73 & 0.000367 \\
\hline Q2FYK6 & Dihydrofolate reductase & FolA & -2.47 & 0.00 \\
\hline Q2FVL9 & Nitrite reductase $[\mathrm{NAD}(\mathrm{P}) \mathrm{H}]$ small subunit & NirD & -2.42 & 0.000192 \\
\hline Q2FYF1 & Cell surface elastin binding & Ebps & -2.32 & 0.001455 \\
\hline Q2FVZ4 & Glycine glycyltransferase & FemX & -2.30 & 0.002388 \\
\hline
\end{tabular}

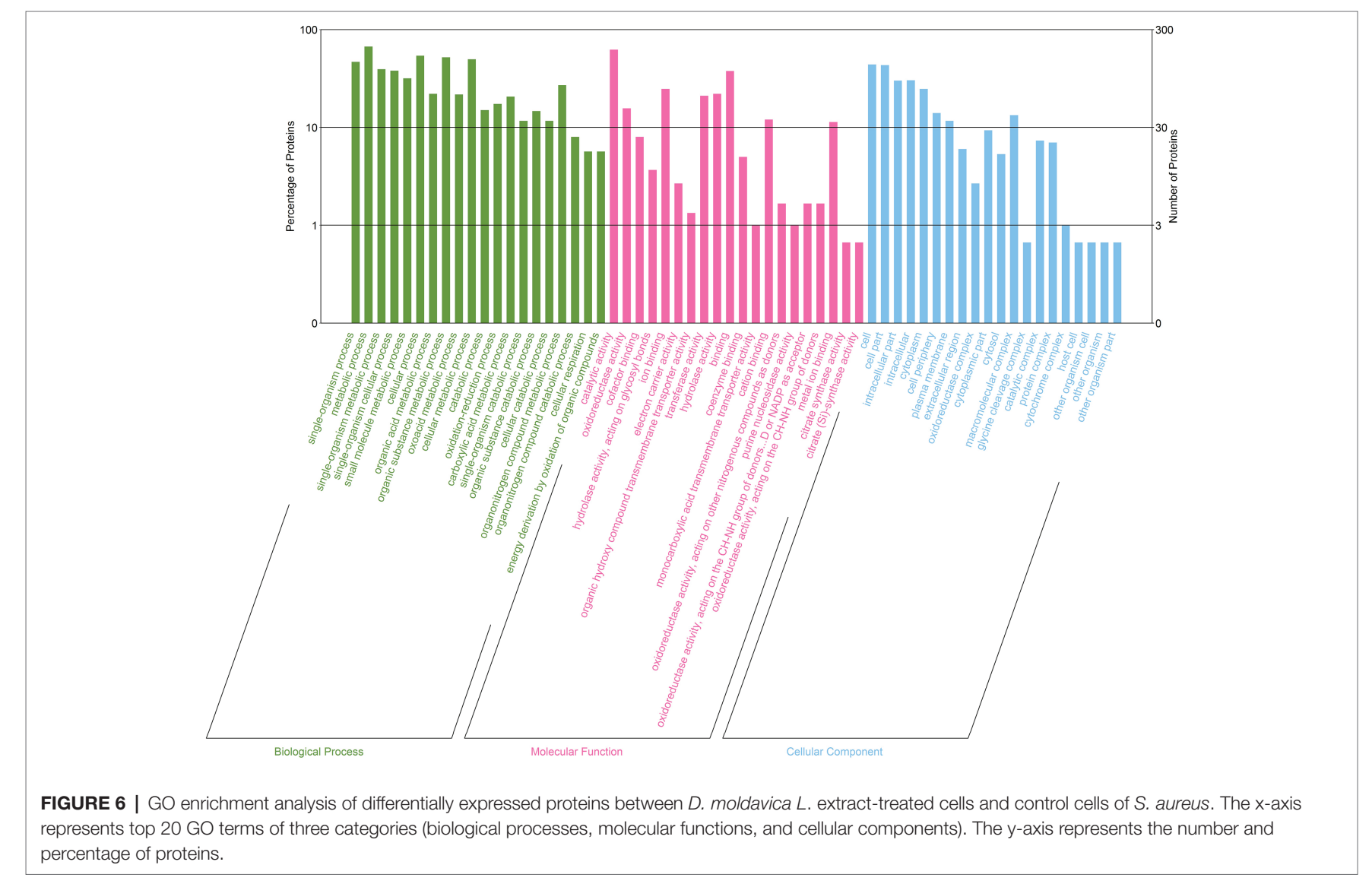

In this study, we showed that tilianin, rosmarinic acid, and unknown compound were the main compounds in the EtOAc fraction of ethanol extract of $D$. moldavica $L$. Therefore, the antibacterial properties against microorganisms of EtOAc fraction can be explained by the antimicrobial activity of its phytoconstituents, such as tannins and rosmarinic acid, which have shown to have effective antibacterial properties (Bigos et al., 2012; Ekambaram et al., 2016).

Changes in the cell morphology of MRSA isolates were detected by scanning electron microscopy analysis. Results 


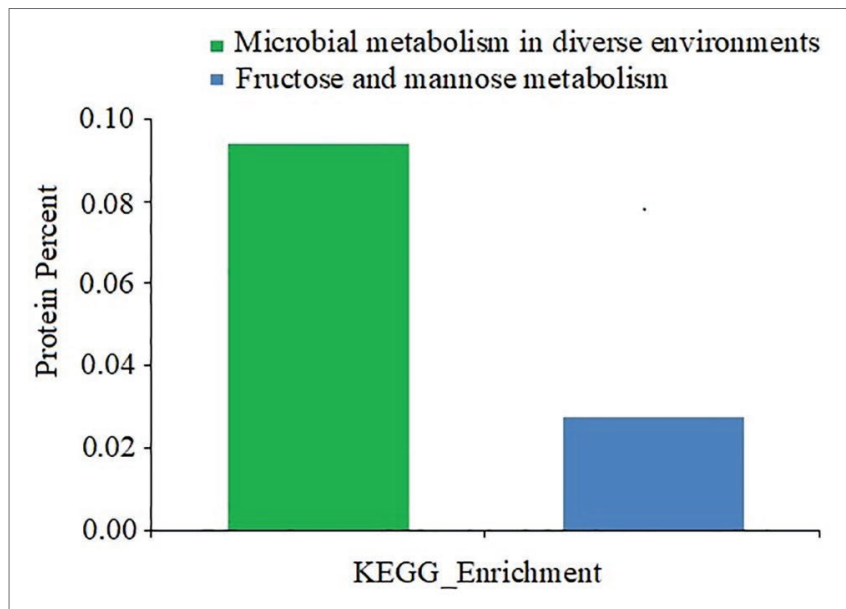

FIGURE 7 | KEGG pathway analysis of differentially expressed proteins between $D$. moldavica $L$. extract-treated cells and control cells of $S$. aureus. The $x$-axis represents different KEGG categories. The $y$-axis represents the number of proteins.

showed that MRSA isolates treated with EtOAc fraction exhibited irregular cell shape and a high degree of cell lysis. These results can be explained by assuming that the EtOAc fraction may damage cell membrane. Moreover, the EtOAc fraction promotes the phosphate ion leakage of MRSA isolates, implicating that this fraction can react with bacterial membrane and increase the membrane permeability. In fact, as already mentioned, extracts of some herbal medicines can damage the phospholipids bilayer in the plasma membrane resulting in leaked cytoplasmic material of the bacteria (Tsuchiya et al., 1996; Maia et al., 2011). Our results seem consistent with previous findings.

We further evaluated the cytotoxicity of the EtOAc fraction against the cell line HaCaT. The cytotoxicity assay showed the cell proliferation inhibition rate of $(33.27 \pm 0.71) \%$ for the EtOAc fraction at high concentration $(100 \mu \mathrm{g} / \mathrm{ml})$. Our results indicated that the treatment of $\mathrm{HaCaT}$ cells with EtOAc fraction did not result in significant cytotoxic effect. We could not find previously published studies on the cytotoxicity of these extracts.

To elucidate the possible molecular mechanism of antibacterial action, we used non-labeled HPLC-MS quantitative proteomic analysis to compare the proteomic pattern of MRSA isolates after exposure to the EtOAc fraction. Several proteins were upregulated that might play a role in the antimicrobial activity of the EtOAc fraction. These proteins include undecaprenyldiphosphatase, delta-hemolysin, and choline dehydrogenase. Undecaprenyl-diphosphatase was involved in peptidoglycan biosynthetic and regulation of cell shape (Wang et al., 2017). Thus, under the burden of severe environment, it may involve in the protective response to external stress to maintain the cell shape. Similarly, glycine betaine is necessary for the survival of bacteria in diverse environment (Yilmaz and Bülow, 2002). Choline dehydrogenase oxidizes choline to betaine aldehyde and then further on to glycine betaine. Upregulated gene expression of glycine betaine biosynthesis-related enzymes induced by stress is clearly observed ( $\mathrm{Xu}$ et al., 2018). Besides the previously reported hemolytic activity, delta-hemolysin is a novel endogenous molecule that inhibits bacterial self-motility due to its detergent effects, which interfere with the hydrophobic nature of $S$. aureus cell surfaces (Omae et al., 2012). Thus, delta-hemolysin might be a target of the EtOAc fraction of $D$. moldavica L. ethanol extract to damage bacterial cell surfaces. In comparison, several proteins were downregulated, including copper chaperone copZ, antiholin-like protein $\operatorname{lrgB}$, and site-specific tyrosine recombinase. The copZ is a key element of copper homeostasis in bacteria. Recent reports showed that copZ may have an independent role in bacterial biofilm formation and competitiveness through novel virulence-regulatory pathways (Garcia et al., 2016). Coincidentally, $\operatorname{lrgB}$ regulates cell envelope and transport related processes (Lama et al., 2012). In S. aureus, $\operatorname{lrgB}$ was shown to encode holin-antiholin-like protein, which was involved in programmed cell death and biofilm formation. Enhanced expression of genes encoding holin-antiholin-like proteins may be one of the main reasons for early biofilm formation (Chen et al., 2015). Moreover, previous studies demonstrated that members of the tyrosine site-specific recombinase family, conserved in microorganism, mediate additional DNA inversions of the genome, which regulates the synthesis of multiple surface proteins (Weinacht et al., 2004). Taken together, those differentially expressed proteins involved in environmental stress response and the regulations of cell surface and biofilm formation.

To understand the gene functions associated with the differentially expressed proteins, the GO enrichment analysis and KEGG pathway analysis were performed. The GO enrichment analysis indicated that the differentially expressed proteins were predominantly metabolism associated. KEGG pathway analysis predicted that the differentially expressed proteins are associated with microbial metabolism in diverse environments as well as fructose and mannose metabolism. In fact, adaption to an environmental stress after treatment of the EtOAc fraction of $D$. moldavica $L$. ethanol extract requires a shift in the expression of proteins involved in specific metabolic pathways. In this study, pyruvate formatelyase activating enzyme, formate acetyltransferase, and glyoxylate reductase were downregulated. These proteins were involved in the anabolic pathways, such as glyoxylate cycle and pyruvate fermentation. However, citrate synthase, FMN reductase, and lactose phosphotransferase system repressor were upregulated. Therefore, our results indicated that the EtOAc fraction of D. moldavica $L$. ethanol extract treatment enhanced aerobic bacterial metabolism and inhibited anaerobic metabolism.

\section{CONCLUSIONS}

We assessed the antimicrobial activity of the EtOAc fraction of D. moldavica $L$. ethanol extract on MRSA strains. No significant cytotoxic effect was detected on HaCaT cell line. We further revealed that this fraction induced the differentially expressed proteins that ultimately lead to downstream consequences resulting in membrane damage, inhibition of biofilm formation and changes in energy metabolism. 


\section{ETHICS STATEMENT}

Ethical approval was obtained from the Second Affiliated Hospital, Baotou Medical College Research and Ethical Review Committee. Informed written consent was obtained from each participant in the study. Any data generated from the specimens protected the patent privacy, confidentiality and anonymity.

\section{AUTHOR CONTRIBUTIONS}

HY and ML experimental strategy, performed experiments and analyses. YL designed and performed experiments. LQ and MJ performed experiments, including the analysis of composition of the D. moldavica ethylacetate extract. ZW conceived this

\section{REFERENCES}

Bigos, M., Wasiela, M., Kalemba, D., and Sienkiewicz, M. (2012). Antimicrobial activity of geranium oil against clinical strains of Staphylococcus aureus. Molecules 17, 10276-10291. doi: 10.3390/molecules170910276

Campanile, F., Bongiorno, D., Borbone, S., and Stefani, S. (2009). Hospitalassociated methicillin-resistant Staphylococcus aureus (HA-MRSA) in Italy. Ann. Clin. Microbiol. Antimicrob. 8:22. doi: 10.1186/1476-0711-8-22

Chen, Y., Gozzi, K., Yan, F., and Chai, Y. (2015). Acetic acid acts as a volatile signal to stimulate bacterial biofilm formation. MBio 6:e00392. doi: 10.1128/ mBio.00392-15

Chen, C. J., and Huang, Y. C. (2014). New epidemiology of Staphylococcus aureus infection in Asia. Clin. Microbiol. Infect. 20, 605-623. doi: 10.1111/1469-0691.12705

Clinical and Laboratory Standards Institute (2018). Performance standards for antimicrobial susceptibility testing, seventeenth informational supplement. (Wayne, PA, USA: Clinical and Laboratory Standards Institute), M100-S17.

Doulgeraki, A. I., Di Ciccio, P., Ianieri, A., and Nychas, G. E. (2017). Methicillinresistant food-related Staphylococcus aureus: a review of current knowledge and biofilm formation for future studies and applications. Res. Microbiol. 168, 1-15. doi: 10.1016/j.resmic.2016.08.001

Ekambaram, S. P., Perumal, S. S., Balakrishnan, A., Marappan, N., Gajendran, S. S., and Viswanathan, V. (2016). Antibacterial synergy between rosmarinic acid and antibiotics against methicillin-resistant Staphylococcus aureus. J. Intercult. Ethnopharmacol. 5, 358-363. doi: 10.5455/jice.20160906035020

Garcia, S. S., Du, Q., and Wu, H. (2016). Streptococcus mutans copper chaperone, CopZ, is critical for biofilm formation and competitiveness. Mol Oral Microbiol 31, 515-525. doi: 10.1111/omi.12150

Jia, P., Xue, Y. J., Duan, X. J., and Shao, S. H. (2011). Effect of cinnamaldehyde on biofilm formation and sarA expression by methicillin-resistant Staphylococcus aureus. Lett. Appl. Microbiol. 53, 409-416. doi: 10.1111/j.1472-765X.2011.03122.x

Jiang, J., Yuan, X., Wang, T., Chen, H., Zhao, H., Yan, X., et al. (2014). Antioxidative and cardioprotective effects of total flavonoids extracted from Dracocephalummoldavica $L$. against acute ischemia/reperfusion-induced myocardial injury in isolated rat heart. Cardiovasc. Toxicol. 14, 74-82. doi: 10.1007/s12012-013-9221-3

Kali, A. (2015). Antibiotics and bioactive natural products in treatment of methicillin resistant Staphylococcus aureus: a brief review. Pharmacogn. Rev. 9, 29-34. doi: 10.4103/0973-7847.156329

Lama, A., Pané-Farré, J., Chon, T., Wiersma, A. M., Sit, C. S., Vederas, J. C., et al. (2012). Response of methicillin-resistant Staphylococcus aureus to amicoumacin A. PLoS One 7:e34037. doi: 10.1371/journal.pone.0034037

Li, G., Shen, M., Yang, Y., Le, S., Li, M., Wang, J., et al. (2018). Adaptation of Pseudomonas aeruginosa to phage $\mathrm{PaP} 1$ predation via o-antigen polymerase mutation. Front. Microbiol. 9:1170. doi: 10.3389/fmicb.2018.01170

Luo, P., Han, X., Wang, Y., Han, M., Shi, H., Liu, N., et al. (2015). Influence of long-term fertilization on soil microbial biomass, dehydrogenase research and wrote the paper. All authors approved the final version of the manuscript.

\section{FUNDING}

This study was supported by the National Natural Science Foundation of China (no. 81660048, 81760056, 81560057). The funder had no role in study design, data collection, analysis and interpretation, or preparation of the manuscript.

\section{ACKNOWLEDGMENTS}

We thank Mr. J. C. Lin of CloudScientific Technology Co., Ltd. for his support during the performance of proteomic analysis.

activity, and bacterial and fungal community structure in a brown soil of northeast China. Ann. Microbiol. 65, 533-542. doi: 10.1007/s13213-014-0889-9

Maia, G. L., Falcão-Silva Vdos, S., Aquino, P. G., de Araújo-Júnior, J. X., Tavares, J. F., da Silva, M. S., et al. (2011). Flavonoids from Praxelis clematidea R.M. King and Robinson modulate bacterial drug resistance. Molecules 16, 4828-4835. doi: 10.3390/molecules 16064828

Martin, K. W., and Ernst, E. (2003). Herbal medicines for treatment of bacterial infections: a review of controlled clinical trials. J. Antimicrob. Chemother. 51, 241-246. doi: 10.1093/jac/dkg087

McDonald, M., Hurse, A., and Sim, K. N. (1981). Methicillin-resistant Staphylococcus aureus bacteraemia. Med. J. Aust. 2, 191-194.

Omae, Y., Sekimizu, K., and Kaito, C. (2012). Inhibition of colony-spreading activity of Staphylococcus aureus by secretion of $\delta$-hemolysin. J. Biol. Chem. 287, 15570-15579. doi: 10.1074/jbc.M112.357848

Oniciuc, E. A., Nicolau, A. I., Hernández, M., and Rodríguez-Lázarod, D. (2017). Presence of methicillin-resistant, Staphylococcus aureus in the food chain. Trends Food Sci. Technol. 61, 49-59. doi: 10.1016/j.tifs.2016.12.002

Sakr, A., Brégeon, F., Mège, J. L., Rolain, J. M., and Blin, O. (2018). Staphylococcus aureus nasal colonization: an update on mechanisms, epidemiology, risk factors, and subsequent infections. Front. Microbiol. 9:2419. doi: 10.3389/fmicb.2018.02419

Suo, B., Yang, H., Wang, Y., Lv, H., Li, Z., Xu, C., et al. (2018). Comparative proteomic and morphological change analyses of Staphylococcus aureus during resuscitation from prolonged freezing. Front. Microbiol. 9:866. doi: 10.3389/ fmicb.2018.00866

Tsuchiya, H., Sato, M., Miyazaki, T., Fujiwara, S., Tanigaki, S., Ohyama, M., et al. (1996). Comparative study on the antibacterial activity of phytochemical flavanones against methicillin-resistant Staphylococcus aureus. J. Ethnopharmacol. 50, 27-34. doi: 10.1016/0378-8741(96)85514-0

Wadhwa, G., Kumar, S., Mittal, V., and Rao, R. (2019). Encapsulation of babchi essential oil into microsponges: physicochemical properties, cytotoxic evaluation and anti-microbial activity. J. Food Drug Anal. 27, 60-70. doi: 10.1016/j. jfda.2018.07.006

Wang, C., Huang, Z., Yu, K., Ding, R., Ye, K., Dai, C., et al. (2017). High-salt diet has a certain impact on protein digestion and gut microbiota: a sequencing and proteome combined study. Front. Microbiol. 8:1838. doi: 10.3389/ fmicb.2017.01838

Weinacht, K. G., Roche, H., Krinos, C. M., Coyne, M. J., Parkhill, J., and Comstock, L. E. (2004). Tyrosine site-specific recombinases mediate DNA inversions affecting the expression of outer surface proteins of Bacteroides fragilis. Mol. Microbiol. 53, 1319-1330. doi: 10.1111/j.1365-2958.2004.04219.x

Xu, Z., Sun, M., Jiang, X., Sun, H., Dang, X., Cong, H., et al. (2018). Glycinebetaine biosynthesis in response to osmotic stress depends on jasmonate signaling in watermelon suspension cells. Front. Plant Sci. 9:1469. doi: 10.3389/fpls.2018.01469

Yilmaz, J. L., and Bülow, L. (2002). Enhanced stress tolerance in Escherichia coli and Nicotiana tabacum expressing a betaine aldehyde dehydrogenase/ choline dehydrogenase fusion protein. Biotechnol. Prog. 18, 1176-1182. doi: $10.1021 / \mathrm{bp} 020057 \mathrm{k}$ 
Zhang, J. L., Yan, R. J., Yu, N., Zhang, X., Chen, D. J., Wu, T., et al. (2018). A new caffeic acid tetramer from the Dracocephalum moldavica $L$. Nat. Prod. Res. 32, 370-373. doi: 10.1080/14786419.2017.1359168

Conflict of Interest Statement: The authors declare that the research was conducted in the absence of any commercial or financial relationships that could be construed as a potential conflict of interest.
Copyright (๑) $2019 \mathrm{Yu}, \mathrm{Liu}, \mathrm{Liu}, \mathrm{Qin}$, Jin and Wang. This is an open-access article distributed under the terms of the Creative Commons Attribution License (CC $B Y)$. The use, distribution or reproduction in other forums is permitted, provided the original author(s) and the copyright owner(s) are credited and that the original publication in this journal is cited, in accordance with accepted academic practice. No use, distribution or reproduction is permitted which does not comply with these terms. 